\title{
Tree Planar Languages
}

\author{
Christophe Costa Florêncio \\ K.U. Leuven \\ Department of Computer Science \\ Celestijnenlaan 200A \\ B-3001 Leuven \\ Belgium \\ Chris.CostaFlorencio@cs.kuleuven.be
}

\begin{abstract}
Planar languages offer an alternative to grammars and automata for representing languages. They are based on hyperplanes in a feature space associated with a string kernel, which corresponds to a set of linear equalities over features.

This makes planar languages inherently learnable, in the sense of being identifiable in the limit from positive data, i.e. learnable in an unsupervised setting, even under strong constraints on the learner's behaviour and on computational resources used.

The position in the Chomsky hierarchy of any class of planar languages depends on the particular kernel it is based on. Even quite simple string kernels generate mildly context-sensitive languages, and so already have enough expressive power to deal with linguistic constructions.

We extend the notion of planar language to tree planar language. The motivation for this is mainly linguistic. These trees could be used to represent derivations, or meaning as logical forms, for example, but there are also applications in bioinformatics or web mining.

In this paper the expressive power of some tree planar languages is analysed and it is shown that some extend beyond the range of regular tree languages. They thus are able to capture all sorts of linguistic phenomena, making them potentially interesting for NLP applications.
\end{abstract}

\section{Kernels and planar languages}

Planar languages, first proposed in [2, 3], offer an alternative to grammars and automata for representing languages. They are defined as sets of strings that map to points in a feature space associated with a string kernel, these points make up a hyperplane. They can be notated in different ways, perhaps the most obvious (and readable) one is as a set of linear equalities over features. Kernels are commonly used in a supervised learning setting, in combination with support vector machines, to learn halfspaces or clusters. The planar language approach deviates from this, in the sense that no negative data is required. It does take full advantage of the benefits that kernels offer, i.e. computational efficiency and modularity. Kernels can be combined at will, and can be used with any (geometric) learning algorithm.

Planar languages form a parameterized family of language classes. The position in the Chomsky hierarchy of any such class from this family depends on the particular kernel it is based on. Since the motivation for planar languages is mainly linguistic, we are especially interested in kernels that generate mildly context-sensitive languages. Even languages based on quite simple 'standard' string kernels, originally intended for use in bioinformatics, turn out to contain such languages. These languages have enough power to handle constructions like crossing dependencies, permutation closed languages like MIX, center embeddings etcetera ([2]).

It has been shown that planar languages are learnable in the sense of being identifiable in the limit from positive data, even under strong constraints on the learner such as strong monotonicity, set-drivenness, small training sets and polynomial update time ([3]). Any class of planar languages is closed under intersection and reversal, but, remarkably, not under union and concatenation.

\subsection{Applications}

In this paper the notion of planar language is extended to tree languages. This is a natural and obvious step both from a linguistic and a practical perspective; being able to obtain some kind of derivation for a given utterance is obviously far more desirable than simply judging its grammaticality. One can for instance think of an application where strings 
are mapped to points in a feature space by a string kernel, a linear map is used to find corresponding points in a feature space associated with a tree planar language, and an inverse function is used to obtain the trees corresponding to these points. These trees might represent derivations, or logical forms, for example. Such a system could be trained with string-tree pairs.

Like many other techniques from the field of grammar induction, potential applications are not limited to natural language processing. Tree languages are also relevant to, for example, the fields of web mining, bioinformatics and computer vision.

\section{Definitions}

\subsection{Planarity}

We will use mainly notation from [3]. Given a particular kernel $\kappa$ and associated feature map $\phi: \Sigma^{*} \rightarrow H$ we can give the following definition:

Definition 2.1 A language $L \subseteq \Sigma^{*}$ is $\kappa$-planar if there is a finite set of strings $\left\{u_{1}, \ldots, u_{n}\right\}$ such that $L=\left\{w \in \Sigma^{*}\right.$ $\left.\exists \alpha_{1} \ldots \alpha_{n} \in \mathbb{R}: \sum_{i=1}^{n} \alpha_{i}=1 \wedge \sum_{i=1}^{n} \alpha_{i} \phi\left(u_{i}\right)=\phi(w)\right\}$.

Given a $\kappa$-planar language $L$ we can define the rank of $L$ to be the cardinality of a minimal subset $U \subseteq L$ that defines the language.

Note that this definition restricts planar languages to those of finite rank, and secondly that we restrict ourselves to affine combinations of strings $u_{i}$.

Definition 2.2 For a language $L \subseteq \Sigma^{*}$, we define $\mathrm{H}(L)=$

$$
\begin{aligned}
\{h \in H \mid & \exists n>0, w_{1} \ldots w_{n} \in L, \alpha_{1}, \ldots \alpha_{n} \text { such that } \\
& \left.\sum_{i=1}^{n} \alpha_{i}=1, \sum_{i=1}^{n} \alpha_{i} \phi\left(w_{i}\right)=h\right\}
\end{aligned}
$$

where $H$ is the feature space. Thus $\mathrm{H}(L)$ is the smallest hyperplane that contains the image of $L$.

For any finite set of strings $U=\left\{u_{1}, \ldots, u_{n}\right\}$, test string $v$, and any polynomially evaluable kernel $\kappa$ with associated feature map $\phi$, there is an algorithm for deciding whether $\phi(v) \in \mathrm{H}\left(u_{1}, \ldots, u_{n}\right)$, which runs in time polynomial in $n,|v|$ and $\sum_{i}\left|u_{i}\right|$. This algorithm is described in [10] and is based on standard mathematical techniques. It involves computations using only the matrix of kernel values. It then normalises this matrix, which has the effect of translating the data in feature space so that the mean of the data lies in the origin, and performs an eigendecomposition of this matrix. It is then easy to project the test point $v$ onto the perpendicular vectors to compute the (Euclidean) distance in feature space of the point from the hyperplane spanned by $U$.

\subsection{Trees}

First we give some standard definitions concerning trees:

Definition 2.3 $A$ tree $T$ is a directed connected acyclic graph in which each node $n$ has in-degree $d^{-}(n)=1$, except the root $r(T)$ which has in-degree 0.

Nodes with out-degree $d^{+}(n)=0$ are called leaf nodes or leaves, nodes with out-degree $d^{+}(n)>0$ are called internal nodes.

The nodes directly reachable from an internal node $n$ are called its children, $n$ is their parent. Two children of a single parent are said to be siblings.

The nodes reachable from a given node $n$ are its descendants, $n$ is called their ancestor.

The depth of a node is the length of the path from the root to the node (number of edges).

The height of a tree $T$ is the maximum of the depths of leaves in $T$.

A proper tree is a tree that contains at least one edge.

A structured (ordered) tree has a fixed order over the parent-child relation, given by $\operatorname{ch}_{1}(n), \ldots, \operatorname{ch}_{d^{+}(n)}(n)$.

A $k$-ary tree is a tree such that the out-degrees of all its nodes is bounded by $k$. In the case of $k=2$, such a tree is called a binary tree. In the case that every node has an out-degree of either 0 or 2, such a tree is also a full binary tree.

$A$ labelled tree is a tree in which each node is associated with a label. The set of all proper trees is denoted by $\mathcal{T}$, the set of all proper trees with labels from $A$ by $\mathcal{T}_{A}$.

One can think of the following as the tree analogues of prefixes, suffixes and substrings:

Definition 2.4 A complete subtree $\tau(n)$ of a tree $T$ at node $n$ is the tree that has $n$ as its root and is composed of all and only the descendants of $n$, and all edges leading out from $n$ and its descendants.

A co-rooted subtree of a tree $T$ is the tree obtained by removing the complete subtrees of all children of some set of internal nodes.

A general subtree of a tree $T$ is any co-rooted subtree of a complete subtree of $T$.

We will use $n_{i}(T) \leq n_{j}(T)$ to denote that in tree $T$, the node indexed by $j$ is an ancestor of the node indexed by $i$, in the case of a proper ancestor we will use $<$.

The least (or nearest) common ancestor of $n_{i}$ and $n_{j}$ is denoted by $n_{i} \smile n_{j}$.

Similarly, $n_{i}(T) \prec n_{j}(T)$ is used to denote that in tree $T$, the node indexed by $i$ is to the left of the node indexed by $j$.

An elastic subtree is said to occur in tree $T$ if it is embedded in $T$ while respecting ancestor and sibling relationships. More formally: 
Definition 2.5 An elastic subtree $S$ of proper tree $T$ is a tree such that

1. $S$ is a proper tree,

2. there exists a mapping $\psi$ from indices over nodes of $S$ to indices over nodes of $T$ such that the ancestordescendent and sibling order in $T$ is respected, i.e.:

(a) for all i for which $n_{i}(S)$ is defined, $\operatorname{Label}\left(n_{i}(S)\right)=\operatorname{Label}\left(n_{\psi(i)}(T)\right)$,

(b) if $n_{i}(S) \leq n_{j}(S)$, then $n_{\psi(i)}(T) \leq n_{\psi(j)}(T)$,

(c) if $n_{i}(T) \leq n_{j}(T)$, then $n_{\psi^{-1}(i)}(S) \leq$ $n_{\psi^{-1}(j)}(S)$, if $\psi^{-1}(i)$ and $\psi^{-1}(j)$ exist,

(d) if $n_{i}(S) \prec n_{j}(S)$, then $n_{\psi(i)}(T) \prec n_{\psi(j)}(T)$,

(e) if $n_{i}(T) \prec n_{j}(T)$, then $n_{\psi^{-1}(i)}(S) \prec$ $n_{\psi^{-1}(j)}(S)$, if $\psi^{-1}(i)$ and $\psi^{-1}(j)$ exist.

This notion is closely related to the Tai mapping ([11]), which was defined in the context of tree-edit distance. It is also related to the notion of alignment of trees. See [7] and [8] for a detailed discussion. An efficient algorithm for computing edit distance for ordered trees can be found in [1].

In the rest of the paper, trees will be written as terms of the form $\left(R, C_{1}, \ldots, C_{n}\right)$, where $\mathrm{R}$ is the label of the root, and $C_{1} \ldots C_{n}$ are its children. These are either labels of the child nodes, or are themselves trees.

\section{Tree kernels}

If one views tree kernels as generalizations of string kernels, the obvious first step is to consider the tree planar language based on the generalization of the simplest possible string kernel. This is the Parikh kernel, which is based simply on the counts of occurrences of symbols in a given string. The analogous tree kernel is based just on counts of labels of the nodes and ignores structure. This tree kernel is known as the Bags of Labels (Bol) kernel ([7]), and is of little interest, other than providing a baseline for experimentally validating tree kernels. The class of Parikh-tree planar languages thus consists simply of all tree languages definable by linear equalities over occurrences of node labels.

Before we discuss more involved tree kernels, we discuss the simpler occurrence-based tree kernels.

\subsection{Occurrence-based tree kernels}

We deem a (tree) kernel occurrence-based if the values in the feature vector obtained from its corresponding embedding are boolean and are assigned according to whether a given subtree occurs in the trees that are being compared.

\subsubsection{The co-rooted subtree kernel}

Consider the following definition taken from [10]:

Definition 3.1 The feature space associated with the corooted subtree kernel is indexed by $I=\mathcal{T}$, the set of all proper trees, with the embedding given by

$$
\phi_{S}^{r}(T)=\left\{\begin{array}{l}
1 \text { if } S \text { is a co-rooted subtree of } T \\
0 \text { otherwise. }
\end{array}\right.
$$

The associated kernel is defined as

$$
\kappa_{r}\left(T_{1}, T_{2}\right)=\left\langle\phi^{r}\left(T_{1}\right), \phi^{r}\left(T_{2}\right)\right\rangle=\sum_{S \in \mathcal{T}} \phi_{S}^{r}\left(T_{1}\right), \phi_{S}^{r}\left(T_{2}\right) .
$$

Note that the embedding is injective: since the largest corooted subtree of $T$ is $T$ itself, the inverse function exists.

\subsubsection{The all-subtree kernel}

The definition of the all-subtree kernel is also taken from [10]:

Definition 3.2 The feature space associated with the allsubtree kernel is indexed by $I=\mathcal{T}$, the set of all proper trees, with the embedding given by

$$
\phi_{S}(T)=\left\{\begin{array}{l}
1 \text { if } S \text { is a subtree of } T ; \\
0 \text { otherwise. }
\end{array}\right.
$$

The associated kernel is defined as

$$
\kappa\left(T_{1}, T_{2}\right)=\left\langle\phi\left(T_{1}\right), \phi\left(T_{2}\right)\right\rangle=\sum_{S \in \mathcal{T}} \phi_{S}\left(T_{1}\right), \phi_{S}\left(T_{2}\right) .
$$

Note that this embedding is again injective, since the largest subtree of $T$ is $T$ itself.

\subsubsection{Occurrence-based tree kernels: their associated planar languages}

It is easy to see that both the co-rooted and all-subtree planar languages form the class of all finite tree languages. This class is of course completely trivial, but there is at least one obvious way of deriving more interesting classes from them: bounding the size of the subtrees that are considered by the kernels.

Analogous to the string kernel case we can define the $k$-co-rooted subtree kernel and $k$-subtree kernel where $k$ specifies the size (height) of the subtrees considered. The corresponding embeddings are not injective, but the size constraints restrict the corresponding classes of planar languages in a way that makes them more interesting in terms of expressive power. 
Let $S$ be a finite set of trees of height $k$. It is easy to see that any $k$-co-rooted planar language consists of the union of just such a set $S$ with the set of all trees for which a tree in $S$ is a co-rooted subtree.

Any $k$-subtree planar language can be defined by $n$ finite sets of trees of height $k, S_{1}$ to $S_{n}$, the corresponding language $L$ is such that for any tree $t$ in $L$, all trees in $S_{i}, 1 \leq i \leq n$ occur as its subtrees.

\subsection{Count-based tree kernels}

As the name implies, count-based kernels are based on counting the number of occurrences of subtrees. Convolution kernels ([6]) offer a general framework for dealing with structured data. A convolution kernel can be defined by specifying decompositions of such structures and computing the products of sub-kernels before summing over these decompositions. This approach allows formulation of tree kernels in the form of recursive definitions. Such definitions suggest naive algorithms that can be implemented very easily. These will generally not be very efficient, however their recursive structure generally permits the use of dynamic programming techniques to speed up certain subcomputations. We will not pursue algorithmic issues further in this paper, see [7] for some examples and pseudocode. We will not use recursive definitions either, since alternatives exist that are conceptually cleaner and more readable.

\subsubsection{The labeled ordered tree kernel}

The labeled ordered tree kernel was first proposed in [7]. It is much like the parse-tree kernel from [5], but their kernel was less flexible, since its use is limited to trees where no node has its label in common with any of its siblings.

Definition 3.3 The feature space associated with the labeled ordered tree kernel is indexed by $I=\mathcal{T}$, the set of all proper trees, with the embedding given by

$$
\phi_{S}(T)=\text { the number of times } S \text { occurs as subtree of } T \text {. }
$$

The associated kernel is defined as

$$
\kappa\left(T_{1}, T_{2}\right)=\left\langle\phi\left(T_{1}\right), \phi\left(T_{2}\right)\right\rangle=\sum_{S \in \mathcal{T}} \phi_{S}\left(T_{1}\right), \phi_{S}\left(T_{2}\right) .
$$

Injectivity is trivial.

The class of labeled ordered tree planar languages is the class of all finite tree languages. If we limit the kernel so that it only considers subtrees of height $k$, we obtain the $k$-labeled ordered tree planar languages. This class contains tree languages reminiscent of derived tree languages of Multi-Component TAG (MCTG), which allow specification of simultaneous adjoining. Given $k=3$, a linear equation like $S_{|(V P, V, N)|}=S_{|(P P, P, N)|}$ states that all occurrences of the former subtree in a given tree must have a matching occurrence of a subtree of the latter form, and vice versa. Such tree languages are obviously not regular.

Apart from considering just subtrees of height $k$, it is also possible to consider just subtrees of height up to $k$, we call the corresponding class the all- $k$-subtree tree planar languages. This class contains context-free tree languages, amongst which is one that is well-known from the TAG literature. This tree language consists of 'fish-bone'like derivations for $a^{n} b^{n} c^{n} d^{n}$, i.e., they take the form of $(S, a,(S, c, \epsilon, d), b),(S, a,(S, a,(S, c,(S, c, \epsilon, d), d), b), b)$ etc. Obviously, the yields of these trees constitute a mildly context-sensitive language. Expressed in the form of linear equations it looks like this:

$$
\begin{gathered}
S_{|(S, a, S, d)|}=S_{|(S, b, S, c)|} \\
S_{|(S, a, S, d)|}=S_{|a|}, S_{|(S, b, S, c)|}=S_{|b|} \\
S_{|(S, a, S, d)|}=S_{|d|}, S_{|(S, b, S, c)|}=S_{|c|} \\
S_{|(S, b,(S, a, S, d), c)|}=0 \\
S_{|(S, a, a, a)|}+S_{|(S, a, a, b)|}+S_{|(S, a, a, \epsilon)|}+\cdots \\
+S_{|(S, a, a, S)|}+S_{|(S, \epsilon, S, S)|}+S_{|(S, S, S, S)|}=S_{|S|} \\
S_{|(S, b, \epsilon, c)|}=1
\end{gathered}
$$

These equations specify the following restrictions:

1. states that the auxiliary subtrees that have $S$ as root, and $a, d$ and $b, c$ as left- and rightmost leaves, respectively, occur equally often,

2. states that such subtrees with $a, b$ as leftmost leaf can only have $d, c$ as rightmost leaf, respectively,

3. states that such subtrees with $c, d$ as rightmost leaf can only have $a, b$ as leftmost leaf, respectively,

4. states that the auxiliary subtrees with $b, c$ as left- and rightmost leaves, respectively, cannot occur above auxiliary subtrees with $a, d$ as left- and rightmost leaves, respectively,

5. states that only $S$ can be a label for an internal node,

6. states that $\epsilon$ occurs once at the bottom of the tree, and nowhere else. 
Note that in order to state these restrictions, counts of several different sizes of subtrees seem to be needed, so this language does not seem to be planar for the $k$-subtree kernel. It is of course possible to define a tree kernel that considers subtrees up to height $k$, analogous to the all- $k$ subsequences string kernel. The tree language specified above is an element of the set of tree planar languages induced by such a kernel.

\subsubsection{The elastic labeled ordered tree kernel}

This kernel is an extension of the labeled ordered tree kernel in the sense that the structures of subtrees are considered elastic. Recall Definition 2.5:

Definition 3.4 The feature space associated with the elastic labeled ordered tree kernel is indexed by $I=\mathcal{T}$, the set of all proper trees, with the embedding given by

$$
\begin{aligned}
& \phi_{S}(T)=\text { the number of times } S \text { appears as elastic subtree } \\
& \quad \text { in } T .
\end{aligned}
$$

The associated kernel is defined as

$$
\kappa\left(T_{1}, T_{2}\right)=\left\langle\phi\left(T_{1}\right), \phi\left(T_{2}\right)\right\rangle=\sum_{S \in \mathcal{T}} \phi_{S}\left(T_{1}\right), \phi_{S}\left(T_{2}\right) .
$$

Note that this kernel is very similar to a kernel discussed in [7], the only difference is that it does not allow label mutations. Injectivity is again trivial.

The class of elastic tree planar languages is equivalent to the class of all finite tree languages. If we limit the kernel so that it only considers subtrees of height $k$, we obtain the $k$-elastic tree planar languages. Intuitively elastic trees are a generalization of non-contiguous substrings, so one would expect this embedding not to be injective, for the same reason that the $k$-substring kernel is not injective ([3]).

This intuition is correct, it is easy to see that the corresponding embedding is not injective. For $k=2$ the unarybranching tree $(a,(b,(b, a)))$ maps to the same point in feature space as $(b,(a,(a, b)))$. This example can be extended to $n$-ary branching trees of the same height, for any given $n$ : any such tree, such that any path from its root to any leaf is equivalent to either of these trees, maps to the same point. This implies that this class does not contain all finite languages.

\subsubsection{The gap-weighted subtree kernel}

The previous tree kernels can be regarded as generalizations of existing string kernels. As far as we are aware, such a generalization has never been proposed for the gapweighted string kernel. This can be done in several different ways, but before we propose one we give the formal definition of this string kernel:
Definition 3.5 The gap-weighted subsequences embedding is based on all non-contiguous subsequences of length $p$ where each occurrence is weighted exponentially by the number of gaps. This is adjusted by a hyper-parameter $\lambda$. $\phi_{u}(s)=\sum_{\mathbf{i}: u=s(\mathbf{i})} \lambda^{l(\mathbf{i})}$ where $|u|=p$. The function $l(i)$ is defined as $1+i_{|i|}-i_{1}$.

The gap-weighted subsequences feature kernel is the kernel based on the gap-weighted subsequences feature map.

Thus, the coordinates will have values based on polynomials over $\lambda$. The intuition behind using an exponential decay value is that the larger the gaps in a subsequence that two strings have in common, the less similar these strings should be considered w.r.t. to that subsequence.

There are also sound theoretical reasons to do this. It has often been noted in the kernel literature that without exponential weighting, one often gets very large values on the diagonal of the Gram matrix. Kernel methods do not perform well in such situations. This is known as the diagonal dominance problem, see [9] for an extensive discussion and a proposed solution.

This embedding is known to be injective, given a nonintegral rational value for $\lambda$ (see [4] for a proof).

Definition 3.6 The feature space associated with the gapweighted subtree kernel is indexed by $I=\mathcal{T}$, the set of all proper trees, with the embedding given by $\phi_{S}^{p}(T)=$

$$
\sum_{\mathbf{i}: u=T(\mathbf{i})} \sum_{a \in \text { paths }(S)} \sum_{b \in \operatorname{paths}(u)} \phi_{a}^{p}(b), \operatorname{dim}(u)=\operatorname{dim}(S)
$$

where paths $(x)$ stands for paths $(x, \operatorname{depth}(S))$. The associated kernel is defined as

$$
\kappa_{p}\left(T_{1}, T_{2}\right)=\left\langle\phi^{p}\left(T_{1}\right), \phi^{p}\left(T_{2}\right)\right\rangle=\sum_{S \in \mathcal{T}} \phi_{S}^{p}\left(T_{1}\right), \phi_{S}^{p}\left(T_{2}\right)
$$

where paths $(S)$ yields the set of all paths from the root to any leaf in $S$, and $\operatorname{depth}(S)$ the length of the longest path in $S$. The function $\operatorname{dim}(S)$ yields the dimensions of tree $S$, $\phi^{p}$ is the embedding underlying the definition of the gapweighted string kernel.The embedding is injective, since the largest gap-weighted subtree of tree $T$ is $T$ itself.

Informally, this kernel treats every distinct path from the root of every elastic subtree of a given tree $T$ as a string $S u b$ to be matched to the corresponding paths from $T$. This matching is performed in the same way as in the gap-weighted string kernel. Note that this kernel does not simply treat all paths in $T$ as strings to be used as input to the gap-weighted string kernel. Such a kernel would treat ordered trees as unordered trees, this kernel takes the order into account. 
For the gap-weighted subtree kernel, we can define languages such as the following: ${ }^{1}$

$$
S_{\left|\left(a,,_{-}, b,-,,_{-}\right)\right|}=3 \lambda^{4}
$$

Informally, this corresponds to defining a language consisting of just all trees containing exactly 3 subtrees that have $a$ as root and $b$ as leftmost leaf, and have height 3 . Furthermore, for no two nodes $n_{1}$ and $n_{2}$ from two distinct subtrees, $n_{1}<n_{2}$, i.e., for any node from such a subtree, there is no node from another such subtree that is on the same path. This is not the only type of language in this class, currently we do not have a full characterization.

\subsection{Roots and leaves}

Under certain circumstances it may be desirable to assign some special status to the root and leave nodes of trees. Analogous to the case of string languages, this can be done by making the root a direct descendant of a node marked with the special label \#, and attach a node with the same label to every leaf. If the resulting tree has to be binary, we can of course attach extra nodes such that the original root now has a (left) sibling marked \#, and all the original leaves have two descendants with that label.

In combination with any of the counting tree kernels considered in this paper it is possible to for example restrict the labels on the leaves to a subset of all labels used in a given tree language. This is obviously useful in a linguistic context.

\section{Conclusion}

We have shown that the notion of planar string language can be extended to tree planar language in a sensible way. The resulting language classes have high expressive power and are efficiently learnable from a small dataset. Our approach therefore has potential for applications in NLP, especially in the context of annotated corpora.

One drawback is that the learning algorithm from $[2,3]$ is noise-sensitive. Given the modularity of kernel-based methods, there is no problem at all with replacing it, but whether a given learning algorithm is more robust to noise depends partly on the language class, and thus on the kernel. It also remains to be seen just how efficient a more robust learning algorithm can be.

The tree kernels considered here are just a sample from the existing literature. Many others exist, and it is generally not that hard to come up with new ones that are designed for use in a specific field. It might be interesting from a theoretical viewpoint to see what tree language is planar for

\footnotetext{
${ }^{1}$ Note the similarity to the (string) schema kernel, and its relation to the gap-weighted kernel.
}

a tree kernel based on, for example, the less-constrained tree mapping, or the closest ancestor operator $A \smile B=c$.

Acknowledgements Most of the research reported in this paper was carried out during a 2-month visit to the $\mathrm{Lab}$. Hubert Curien, Univ. Jean Monnet, St Etienne, France, funded by the IST Programme of the EU, under the PASCAL Network of Excellence. I would also like to thank Colin de la Higuera for stimulating discussions.

\section{References}

[1] W. Chen. New algorithm for ordered tree-to-tree correction problem. Journal of Algorithms, 40(2):135-158, August 2001.

[2] A. Clark, C. Costa Florêncio, and C. Watkins. Languages as hyperplanes: grammatical inference with string kernels. In ECML, 17th European Conference on Machine Learning. Springer-Verlag, 2006.

[3] A. Clark, C. Costa Florêncio, C. Watkins, and M. Serayet. Planar languages and learnability. In International Colloquium on Grammatical Inference (ICGI), Tokyo, 2006.

[4] A. Clark and C. Watkins. Some alternatives to Parikh matrices using string kernels, To appear.

[5] M. Collins and N. Duffy. Convolution kernels for natural language. In Proceedings of the 14th Conference on Neural Information Processing Systems, 2001.

[6] D. Haussler. Convolution kernels on discrete structures. Technical Report UCS-CRL-99-10, UC Santa Cruz, 1999.

[7] H. Kashima and T. Koyanagi. Kernels for semi-structured data. In ICML '02: Proceedings of the Nineteenth International Conference on Machine Learning, pages 291-298, San Francisco, CA, USA, 2002. Morgan Kaufmann Publishers Inc.

[8] T. Kuboyama, K. Shin, and H. Kashima. Flexible tree kernels based on counting the number of tree mappings. In ECML/PKDD Workshop on Mining and Learning with Graphs, 2006.

[9] B. Schölkopf, J. Weston, E. Eskin, C. Leslie, and W. S. Noble. A kernel approach for learning from almost orthogonal patterns. In ECML '02: Proceedings of the 13th European Conference on Machine Learning, pages 511-528, London, UK, 2002. Springer-Verlag.

[10] J. Shawe-Taylor and N. Christianini. Kernel Methods for Pattern Analysis. Cambridge University Press, 2004.

[11] K.-C. Tai. The tree-to-tree correction problem. J. ACM, 26(3):422-433, 1979. 